\title{
FIB Sample Preparation for In Depth EDS Analysis
}

\author{
Roberto Garcia ${ }^{1}$, Fred A. Stevie ${ }^{1^{*}}$ and Lucille Giannuzzi ${ }^{2}$ \\ 1. Analytical Instrumentation Facility, North Carolina State University, Raleigh, NC, USA. \\ 2. EXpressLO LLC, Lehigh Acres, FL, USA. \\ * Corresponding author: fred_stevie@ncsu.edu
}

The FIB has been extensively used to prepare specimens for many analytical techniques [1,2]. FIB instruments are typically paired with an SEM column but lack elemental analysis capability. AES, XPS, SIMS, and EDS are the most common techniques for elemental analysis and EDS is probably the easiest to add to an FIB platform. An issue with EDS is the ability to provide in-depth analysis. The EDS analyzed region can be micrometers for a $30 \mathrm{keV}$ electron beam. It is possible to prepare a lift out specimen and then perform a line scan with EDS, [3] but not everyone has lift out capability. Knowledge of the penetration range of the electron beam coupled with material removal using the ion beam can provide significant information about the specimen. Earlier work showed that removal of material behind the region of interest could improve EDS image quality. [4] Cross sections can also be prepared and then studied with EDS. However, the tilted geometry of a FIB section can make interpretation difficult.

A commonly available pre-programmed FIB sample preparation for SEM analysis is a "stair-step" type cut as diagrammed in Fig. 1. With EDS analysis at each step (typically five steps), one can have the result of EDS analysis at five depths into the sample. For $3 \mu \mathrm{m}$ deep steps, we would have a $15 \mu \mathrm{m}$ range of analysis. Excitation of the x-ray lines of many of the elements of interest requires on the order of 10 to $15 \mathrm{keV}$ primary electrons. A typical penetration depth for $\mathrm{Al}$ at $10 \mathrm{keV}$ is $\sim 700 \mathrm{~nm}$.

Analyses were performed using a FEI Quanta 3D which has electron and FIB columns. Stair step cuts to a depth of $1.5 \mu \mathrm{m}$ were made with $7 \mathrm{nA} 30 \mathrm{keV} \mathrm{Ga}^{+}$over a $20 \mu \mathrm{m}$ x $40 \mu \mathrm{m}$ area with the pattern rotated $90^{\circ}$. The longer dimension was chosen so that a line scan could be taken across the 5 steps, each $8 \mu \mathrm{m}$ wide, and the specimen surface. Samples with approximately $1 \mu \mathrm{m}$ thick layers of Al, TiW, Au, and Cu on Si substrates were analyzed with an Oxford EDS detector and SEM operating at $5.7 \mathrm{nA}$ and 10 or 15 $\mathrm{keV}$. Figure 2 shows the vertical stair step cuts and the location of the line scan for the Al sample which has $0.4 \mu \mathrm{m} \mathrm{SiO}_{2}$ under the Al. Figure 3 shows the EDS line scan results. The Al layer does not sputter evenly but the $\mathrm{Al}, \mathrm{SiO}_{2}$ and $\mathrm{Si}$ can be clearly identified. More complex structures are being investigated to help determine the depth resolution that can be obtained.

The cuts for this study only required about 5 minutes, so this is a rapid method. The introduction of plasma FIB sources that have micro-ampere current capability makes possible the removal of much larger quantities of material. The removal rate can be 50 times faster than with a conventional nanoampere gallium source and would permit much greater depths for this analysis.

\section{References:}

[1] "Introduction to Focused Ion Beams", ed. LA Giannuzzi and FA Stevie (Springer, NY).

[2] FA Stevie, CB Vartuli, LA Giannuzzi, TL Shofner, SR Brown, B Rossie, F Hillion, RH Mills, M Antonell, RB Irwin, and BM Purcell, Surface and Interface Analysis 31 (2001), p. 345-351. 
[3] R Garcia, Y Liu, FA Stevie, Microsc. Microanal. 22 (Suppl 3) (2016) p. 138-139.

[4] CB Vartuli, FA Stevie, JB Bindell, TL Shofner, and BM Purcell, Microscopy and Microanalysis Proceedings 5, Supplement 2 (1999) p. 896-897.

[5] This work was performed in part at the Analytical Instrumentation Facility (AIF) at North Carolina State University, which is supported by the State of North Carolina and the National Science Foundation (award number ECCS-1542015). The AIF is a member of the North Carolina Research Triangle Nanotechnology Network (RTNN), a site in the National Nanotechnology Coordinated Infrastructure (NNCI).

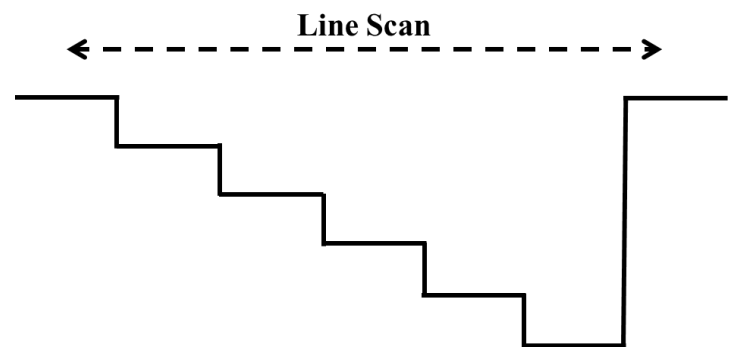

Figure 1. Schematic drawing of FIB sample preparation.

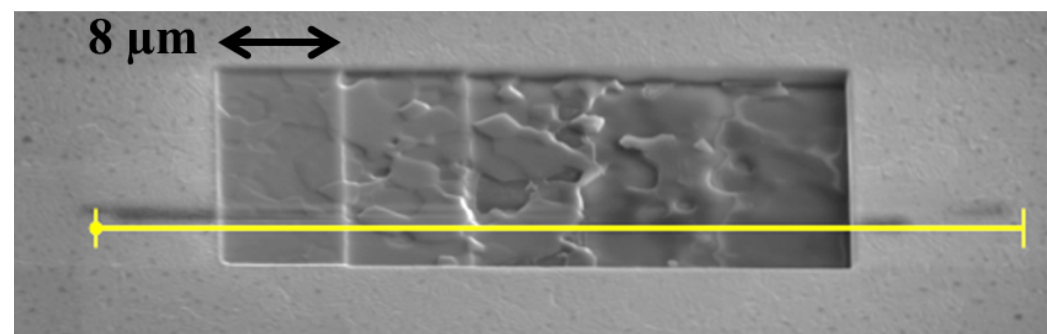

Figure 2. SEM image of Al sample with vertical cuts and location of line scan

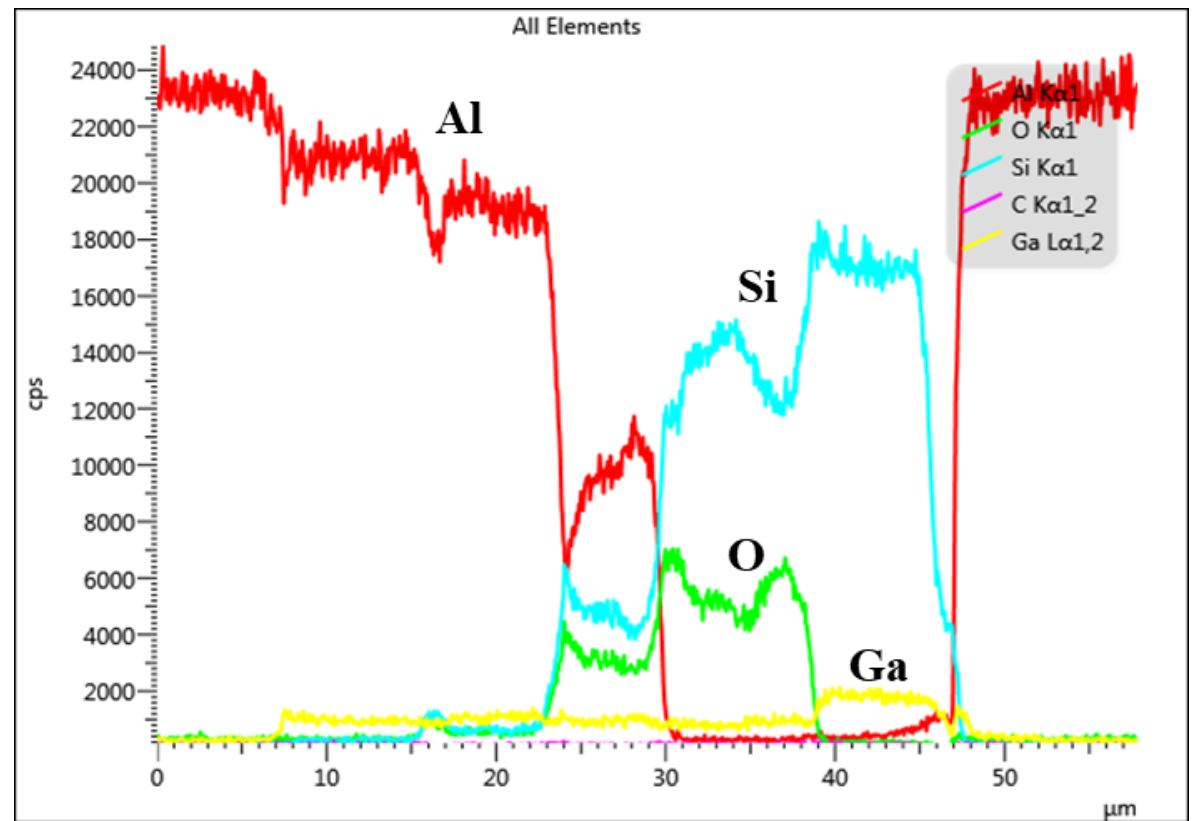

Figure 3. EDS line scan across the stair step FIB cut. 